\title{
Prevalence of depression and its impact on quality of life in frontline otorhinolaryngology nurses during the COVID-19 pandemic in China
}

\author{
Zi-Rong Tian Equal first author, ${ }^{1}$, Xiaomeng Xie ${ }^{\text {Equal first author, } 2,3}{ }^{3}$, Xiu-Ya $\mathbf{L i}^{4}$, Yue Li ${ }^{1}$, Qinge Zhang ${ }^{5}$, Yan-Jie Zhao ${ }^{2,3}$, Teris \\ Cheung ${ }^{6}$, Gabor S. Ungvari ${ }^{7,8}$, Feng-Rong An ${ }^{\text {Corresp., } 5}$, Yu-Tao Xiang ${ }^{\text {Corresp. 2, } 3}$

Objective: Exposure to the coronavirus disease 2019 (COVID-19) was associated with high risk of mental health problems among frontline nurses. This study examined the prevalence of depressive symptoms (depression hereafter) and its impact on quality of life (QOL) in otorhinolaryngology (ENT) nurses during the COVID-19 pandemic in China.Methods: An online study was conducted between March 15 and March 20, 2020. Depression and QOL were assessed using standardized instruments.Results: A total of 1,757 participants were recruited. The prevalence of depression was 33.75\% (95\% Cl: $31.59 \%-35.97 \%)$. Results emerging from multiple logistic regression analysis showed that direct care of COVID-19 patients (OR: $1.440,95 \% \mathrm{Cl}: 1.031-2.012, P=0.032$ ), and current smoking (OR: $3.143,95 \% \mathrm{Cl}: 1.020-9.690, P=0.046)$ were significantly associated with depression. After controlling for covariates, ENT nurses with depression had a lower overall QOL compared to those without depression $\left(F_{(1,1757)}=536.80, P<0.001\right)$. Conclusions:

Depression was common among ENT nurses during the COVID-19 pandemic in China. Considering the negative impact of depression on QOL and care quality, regular screening for depressionshould be conducted in ENT nurses and treatment should be provided. 
Tables: 2

\section{Prevalence of depression and its impact on quality of life in}

6 frontline otorhinolaryngology nurses during the COVID-19

9 1\#Zi-Rong Tian, MN

2,3,\#Xiaomeng Xie, PhD

$11{ }^{4}$ Xiu-Ya Li, BN

12 'Yue Li, MN

$13{ }^{5}$ Qinge Zhang, MD

14 2,3 Yan-Jie Zhao, PhD

$15{ }^{6}$ Teris Cheung, PhD

$16 \quad 7,8$ Gabor S. Ungvari, MD, PhD

$17{ }^{5 *}$ Feng-Rong An, MN

$182^{2,3 *}$ Yu-Tao Xiang, MD, PhD

1. Department of Nursing, Beijing Tongren Hospital, Capital Medical University, Beijing, China

2. Centre for Cognitive and Brain Sciences, University of Macau, Macao SAR, China;

3. Institute of Advanced Studies in Humanities and Social Sciences, University of Macau, Macao

6. School of Nursing, Hong Kong Polytechnic University, Hong Kong SAR, China;

4. Department of Otorhinolaryngology, Beijing Tongren Hospital, Capital Medical University, Beijing, China;

5. The National Clinical Research Center for Mental Disorders \& Beijing Key Laboratory of Mental Disorders Beijing Anding Hospital \& the Advanced Innovation Center for Human Brain Protection, Capital Medical University, School of Mental Health, Beijing, China;

7. Division of Psychiatry, School of Medicine, University of Western Australia / Graylands Hospital, Perth, Australia;

8. University of Notre Dame Australia, Fremantle, Australia;

\# These authors contributed equally to the work. 
36 * Address correspondence to Dr. Yu-Tao Xiang, 3/F, Building E12, Faculty of Health Sciences, 37 University of Macau, Avenida da Universidade, Taipa, Macau SAR, China. Fax: +853-2288-2314;

38 Phone: +853-8822-4223; E-mail: YTXiang@um.edu.mo; and Dr. Feng-Rong An, Beijing Anding

39 Hospital; E-mail: afrylm@sina.com

40 


\section{Abstract}

42 Objective: Exposure to the coronavirus disease 2019 (COVID-19) was

43 associated with high risk of mental health problems among frontline nurses.

44 This study examined the prevalence of depressive symptoms (depression

45 hereafter) and its impact on quality of life (QOL) in otorhinolaryngology (ENT)

46 nurses during the COVID-19 pandemic in China.

47 Methods: An online study was conducted between March 15 and March 20, 48 2020. Depression and QOL were assessed using standardized instruments.

49 Results: A total of 1,757 participants were recruited. The prevalence of 50 depression was 33.75\% (95\% CI: 31.59\%-35.97\%). Results emerging from

51 multiple logistic regression analysis showed that direct care of COVID-19 52 patients (OR: $1.440,95 \% \mathrm{CI}: 1.031-2.012, P=0.032$ ), and current smoking (OR: $3.143,95 \% \mathrm{CI}: 1.020-9.690, P=0.046$ ) were significantly associated with depression. After controlling for covariates, ENT nurses with depression had a lower overall QOL compared to those without depression $\left(F_{(1,1757)}\right.$ $56=536.80, P<0.001)$.

57 Conclusions: Depression was common among ENT nurses during the COVID5819 pandemic in China. Considering the negative impact of depression on QOL and care quality, regular screening for depression should be conducted in ENT nurses and treatment should be provided.

61 Keywords: COVID-19, depression, otorhinolaryngology, quality of life, nurse 


\section{Introduction}

66 The novel coronavirus disease (COVID-19) was first reported in Wuhan, China

67 at the end of 2019. Since then the disease has been reported in more than 68200 countries and territories, and COVID-19 has been declared a global public 69 health emergency (World Health Organization, 2020). The reproduction 70 number of COVID-19 ranges from 2.24 (95\%CI: $1.96-2.55)$ to 3.58 (95\%CI: 71 2.89-4.39) (Zhao et al., 2020). Similar to other respiratory viruses, this virus 72 is spread maqinly by respiratory droplets of infected cases when people speak, 73 cough, or sneeze. In early phase of the COVID-19 outbreak, it was presumed 74 that nosocomial transmission contributed to $41.3 \%$ of the infected patients in 75 the general population and $29 \%$ of infected health care workers (Wang et al., 76 2020). By the nature of the clinical specialty, healthcare workers in otorhinolaryngology (ENT) units have a much higher likelihood to have direct contacts with COVID-19 patients compared with their counterparts in other clinical specialties. ENT nurses are exceptionally susceptible to aerosolized viral particles and high viral loads in the upper respiratory tract. This possibly explained why many health professionals working in ENT units were infected in the early stage of the COVID-19 outbreak (Lu et al., 2020). For example, in

83 the UK an ENT consultant was the first frontline clinician who died on 30 March 842020 in combating COVID-19 (NHS, 2020). Due to heavy clinical workload and high risk of infection, ENT nurses are more likely to suffer from psychological distress, which could increase the risk of more serious mental health problems, such as depression (Venugopal et al., 2020; Xu et al., 2020).

Depression is associated with a range of negative health outcomes, such as increased risk of suicide, poor care quality and impaired occupational 90 functions (Gao et al., 2019; Knight et al., 2018; Woo et al., 2016). In order

91 to reduce the risk of depression and develop appropriate preventive measures, 92 it is important to understand its epidemiology. Quality of life (QOL) has been 93 a widely used comprehensive health outcome in the past decades. To the best 
94 of our knowledge, there have been no studies examining the epidemiology of

95 depression and its impact on QOL in ENT healthcare workers. Therefore, this

96 study set out to examine the prevalence of depressive symptoms (depression

97 hereafter) and their impact on QOL in frontline ENT nurses in China during the 98 COVID-19 pandemic.

\section{Materials \& Methods}

\section{Setting and sample}

102 This was a cross-sectional, anonymous online survey initiated by the 103 Otolaryngology Branch, Chinese Nursing Association between March 15 and 104 March 20, 2020 in China. Due to logistic reasons and the high risk of cross105 infection, random sampling and face-to-face interviews were prohibited in 106 almost all surveys involving frontline health professionals during the COVID10719 outbreak in China. Similar to other studies (Lai et al., 2020; Zhang et al., 108 2020), snowball sampling was used. The survey was conducted using the 109 WeChat-based Questionnaire Star program. WeChat is a social communication 110 application with over 1 billion users in China including all participants in this 111 study. The Questionnaire Star program that has been widely used in many 112 epidemiological surveys (Li, 2016; Liang and Fan, 2020; Xi, 2017) was 113 employed in this study. To be eligible, participants needed to be: 1) aged 18

114 years or above; 2) frontline nurses working in ENT units during the COVID-19 115 outbreak; 3) able to understand the assessment and provide written informed 116 consent. The research protocol was approved by the Institutional Review 117 Board of Beijing Anding Hospital (2020(10)) and all participating hospitals and 118 universities. All the study procedures were carried out in accordance with 119 relevant guidelines. All participants provided informed consent to participate 120 in the study.

\section{Instruments}


123 Basic socio-demographic and clinical variables, such as gender, age, marital

124 status, education level, years of work experience, living circumstances, rank

125 (junior or senior), hospital setting (primary/secondary vs tertiary hospitals),

126 shift duty requirement, type of the unit (inpatient or outpatient), smoking

127 status, and personal experience with the Severe Acute Respiratory Syndrome

128 (SARS) outbreak on 2003 were collected. Three additional standardized

129 questions with dichotomous response (yes/no) were also asked: 1) whether

130 the participant was directly engaged in clinical services for COVID-19 patients;

131 2) whether they had friends, colleagues, or family members infected with

132 COVID-19; and 3) the number of COVID-19 confirmed cases in the province

133 where they lived.

134 The self-report Chinese version of the Patient Health Questionnaire-9

135 (PHQ-9) was used to measure the severity of depression in the past week.

136 The PHQ-9 was validated in Chinese populations with a sensitivity of 0.89

137 and a specificity of 0.77 (Chen, 2015). Each item was scored from 0 to 3, with

138 the total score of $\geqslant 5$ indicating "depression" (Wittkampf et al., 2007). The

139 total score of "5-9", "10-14", "15-19", and "20-27" indicated "mild

140 depression", "moderate depression", "moderate-to-severe depression", and

141 "severe depression", respectively (Wittkampf et al., 2007).

142 Following the example of previous studies (An et al., 2020; Ma et al.,

143 2020; Wang et al., 2006) QOL was estimated with the first two items on

144 overall QOL of the validated World Health Organization Quality of Life

145 Instrument-Brief Version (WHOQOL-BREF) (Skevington et al., 2004). Higher

146 total scores indicated better QOL. The Chinese version of the WHOQOL-BREF

147 has been validated in Chinese populations (Xia et al., 2012).

149 Data analysis

150 Data were analyzed with the IBM Statistical Package for Social Science 151 (SPSS), software version 24.0. Normality of the data was assessed using 
152 the Kolmogorov-Smirnov test. Comparison between the 'depression' and 'no

153 depression' groups in terms of demographic and clinical characteristics were

154 conducted by chi-square test, two samples independent sample $t$-test and 155 Mann-Whitney U test, as appropriate. QOL was compared between the two 156 groups using analysis of covariance (ANCOVA) after controlling the potentially 157 confounding effects of variables with significant group difference in univariate 158 analyses. The independent demographic and clinical correlates of depression 159 were examined using multiple logistic regression analysis with the "Enter" 160 method with depression as the dependent variable. All variables with a $P$-value 161 of less than 0.1 in univariate analyses were entered as independent variables. 162 A $P$-value of less than 0.05 was considered statistically significant (two-tailed).

\section{Results}

165

166

167

168

169

170

A total of 1,757 frontline ENT nurses (females $n=1,746,99.4 \%$ of the sample) participated in the study. The overall prevalence of depression was $33.75 \%$ (95\% CI: 31.59\%-35.97\%). Among the healthcare workers with probable depression ( $\mathrm{N}=593), 421(24.0 \%)$ reported mild, $116(6.6 \%)$ moderate, 42 $(2.4 \%)$ moderate-to-severe, and $14(0.8 \%)$ severe depression. The mean total score of the PHQ-9 scale was $3.73( \pm 4.43)$ in the whole sample.

Table 1 shows the demographic and clinical characteristics of the whole sample separated by depression. Univariate analyses revealed that direct care with confirmed COVID patients $(P=0.025)$, current smoking $(P=0.033)$, and years of work experience $(P=0.020)$ were significantly associated with depression. After controlling for covariates including looking after infected patients, smoking, work experience, depressed nurses were more likely to have overall lower QOL than those without depression $\left(F_{(1,1757)}=527.94\right.$, $P<0.001)$. Five variables with a $P$-value of $<0.1$ were entered in multiple logistic regression analysis as independent variables including working in tertiary hospitals, nursinginfected patients, smoking, age, and work 
181 experience. Direct care of COVID-19 patients (OR=1.441, $P=0.032$ ) and

182 smoking $(\mathrm{OR}=2.880, P=0.048)$ were independently associated with higher

183 risk of depression (Table 2 ).

184

\section{Discussion}

186 To the best of our knowledge, this was the first study that examined the 187 prevalence, demographic and clinical factors associated with depression in 188 ENT nurses during the COVID-19 pandemic. Other studies have examined the 189 epidemiology of depression in health professionals in China. In the early stage 190 of the COVID-19 outbreak at the end of January 2020, 50.4\% of frontline 191 medical professionals working in Wuhan and the surrounding areas of Hubei 192 province reported depression measured using the PHQ-9 with a cut-off value 193 of 5 (Lai et al., 2020). With the same cut off value in the PHQ-9, the 194 prevalence of depression in healthcare workers in Wuhan was 36.9\% between January 29 and February 4, 2020 (Kang et al., 2020). In contrast, the prevalence of depression in frontline healthcare workers was $12.2 \%$ assessed with the PHQ-4 with a lower cut-off value of 3 from February 19 to March 6, 2020 (Zhang et al., 2020). Findings of the current study (33.75\%; 95\% CI: $31.59 \%-35.97 \%$ ) were similar to those of some (Kang et al., 2020), but not all studies (Lai et al., 2020; Zhang et al., 2020). Due to the use of different measurement tools on depression, direct comparison between these studies should be interpreted with caution.

In ENT units, asymptomatic and pre-symptomatic patients with COVID19 may seek help for anosmia, which is a common symptom of COVID-19 (Hopkins et al., 2020). Examinations of the nasal passages and upper airway, intubation and administration of respiratory treatment can induce cough, nausea, sneezing or vomiting (Lu et al., 2020). The nasal pillow masks for patients with obstructive sleep-apnea may allow airborne virus transmission due to loose sealing (Tran et al., 2012). In the 2003 SARS outbreak, clusters 
210 of nosocomial infections were observed among healthcare workers during

211 airway manipulation (JAMA, 2003). All these factors could increase the 212 likelihood of COVID-19 infection for ENT nurses, leading to common mental 213 health problems, such as depression.

214 Similar to previous findings (Lai et al., 2020; Pan et al., 2020), frontline 215 ENT nurses who provided direct care for COVID-19 patients were more likely 216 to have depression. During the COVID-19 outbreak, ENT nurses had to do 217 shift duty andworked longer hours than usual, which can lead to high level of 218 stress. All health professionals were confined to at least two weeks quarantine 219 after they finished care to COVID-19 patients, which can increase their anxiety 220 and induce guilt feelings due to the social stigma affecting their families, as it 221 happened during theSARS epidemic (Holmes et al., 2020; Li et al., 2020; 222 Nickell et al., 2004; Yip et al., 2010). All these factors could substantially 223 increase the risk of depression. Smoking is associated with higher risk of 224 medical conditions and psychiatric disturbances including depression (Chang 225 et al., 2020; Fluharty et al., 2017; Mathew et al., 2017). This study also found 226 that depressed ENT healthcare workers were more likely to smoke(Nilan et 227 al., 2019; Schneider et al., 2019)

228 According to the distress/protection model of QOL (Voruganti et al., 229 1998), QOL is the result of the interaction between protective (e.g., high self230 esteem and good social support) and distressing factors (e.g., physical and 231 psychological stress). Consistent with previous findings (Benedek et al., 2007; 232 Mammen and Faulkner, 2013; Roche et al., 2020) depressed ENT nurses had 233 a poorer QOL compared to the those without depression in this survey. This 234 could be explained by the negative health outcomes of depression, such as 235 impaired psychosocial functioning and somatic symptoms of fatigue, loss of 236 appetite or weight, and insomnia (Anosike et al., 2020; Malhi and Mann, 2018;

237 Parisi et al., 2014; Rakofsky et al., 2013). 
The strengths of this study include the large sample size and the use of standardized instruments. However, several limitations should be addressed.

240 First, because of the cross-sectional design, the causality between the 241 demographic and clinical variables and depression could not be established.

242 Second, data were collected by online self-report, therefore the the validity of 243 certain variables (e.g., history of psychiatric disorders) could not be tested 244 and recall bias could exist, which is a common limitation in all online surveys.

245 Third, due to logistical reasons, relevant factors related to depression in ENT 246 nurses, such as lifestyle, family support, work load including the number of 247 daily outpatient visits and inpatients in participating hospitals, were not 248 obtained. Forth, due to the lack of rating scales on COVID-19-related 249 experiences in China, participants were asked only using three standardized 250 questions with dichotomous response, similar to previous studies (Forte et al., 251 2020; Zhong et al., 2020). Fifth, the snowball sampling method was used, 252 thus the number of ENT nurses who did not complete the assessment or 253 refused to participate in the study could not be recorded. Finally, this is an 254 anonymous survey, therefore, participants' hospital and personal identifying 255 information was not collected. Sixth, the exclusion of participants with pre256 existing mental health problems could have biased the results to an uncertain 257 extent.

258

\section{Conclusions}

260 Depression was common among ENT nurses during the COVID-19 pandemic 261 in China. Considering the negative impact of depression on their QOL and the 262 quality of care ENT nurses provide, regular screening for depression should be 263 conducted for this particularly vulnerable cohort of health workers coupled 264 with easily available treatment .

\section{Acknowledgements}


267 None.

268

269 References

270

271

272

273

274

275

276

277

278

279

280

281

282

283

284

285

286

287

288

289

290

291

292

293

294

295

296

297

298

299

300

301

302

An, Y., Yang, Y., Wang, A., Li, Y., Zhang, Q., Cheung, T., Ungvari, G.S., Qin, M.-Z., An, F.-R., Xiang, Y.-T., 2020. Prevalence of depression and its impact on quality of life among frontline nurses in emergency departments during the COVID-19 outbreak. Journal of Affective Disorders 276, 312-315.

Anosike, C., Isah, A., Igboeli, N.U., 2020. Development and validation of a questionnaire for evaluating knowledge of risk factors for teen depression among health care trainees of a Nigerian university. Asia-Pacific Psychiatry, e12391.

Benedek, D.M., Fullerton, C., Ursano, R.J., 2007. First responders: mental health consequences of natural and human-made disasters for public health and public safety workers. Annu Rev Public Health 28, 55-68.

Chang, E.T., Lau, E.C., Moolgavkar, S.H., 2020. Smoking, air pollution, and lung cancer risk in the Nurses' Health Study cohort: time-dependent confounding and effect modification. Crit. Rev. Toxicol., 1-12.

Chen, M., Sheng, L., Qu, s., 2015. Diagnostic test of screening depressive disorder in general hospital with the Patient Health Questionnaire (in Chinese). Chinese Mental Health 29(4), 241-245.

Fluharty, M., Taylor, A.E., Grabski, M., Munafo, M.R., 2017. The Association of Cigarette Smoking With Depression and Anxiety: A Systematic Review. Nicotine \& tobacco research : official journal of the Society for Research on Nicotine and Tobacco 19(1), 3-13.

Forte, G., Favieri, F., Tambelli, R., Casagrande, M., 2020. COVID-19 Pandemic in the Italian Population: Validation of a Post-Traumatic Stress Disorder Questionnaire and Prevalence of PTSD Symptomatology. Int J Environ Res Public Health 17(11).

Gao, K., Su, M., Sweet, J., Calabrese, J.R., 2019. Correlation between depression/anxiety symptom severity and quality of life in patients with major depressive disorder or bipolar disorder. J. Affect. Disord. 244, 9-15.

Holmes, E.A., O'Connor, R.C., Perry, V.H., Tracey, I., Wessely, S., Arseneault, L., Ballard, C., Christensen, H., Cohen Silver, R., Everall, I., Ford, T., John, A., Kabir, T., King, K., Madan, I., Michie, S., Przybylski, A.K., Shafran, R., Sweeney, A., Worthman, C.M., Yardley, L., Cowan, K., Cope, C., Hotopf, M., Bullmore, E., 2020. Multidisciplinary research priorities for the COVID-19 pandemic: a call for action for mental health science. The Lancet Psychiatry 7(6), 547-560.

Hopkins, C., Surda, P., Kumar, N., 2020. Presentation of new onset anosmia during the COVID-19 pandemic. Rhinology.

JAMA, 2003. Cluster of Severe Acute Respiratory Syndrome Cases Among Protected Health-Care WorkersToronto, Canada, April 2003. JAMA 289(21), 2788-2789.

Kang, L., Ma, S., Chen, M., Yang, J., Wang, Y., Li, R., Yao, L., Bai, H., Cai, Z., Xiang Yang, B., Hu, S., Zhang, K., Wang, G., Ma, C., Liu, Z., 2020. Impact on mental health and perceptions of psychological care among medical and nursing staff in Wuhan during the 2019 novel coronavirus disease outbreak: A cross-sectional study. Brain Behav Immun. 
303 Knight, M.J., Air, T., Baune, B.T., 2018. The role of cognitive impairment in psychosocial functioning in remitted

304 depression. J. Affect. Disord. 235, 129-134.

305 Lai, J., Ma, S., Wang, Y., Cai, Z., Hu, J., Wei, N., Wu, J., Du, H., Chen, T., Li, R., Tan, H., Kang, L., Yao, L.,

306 Huang, M., Wang, H., Wang, G., Liu, Z., Hu, S., 2020. Factors Associated With Mental Health Outcomes Among

307 Health Care Workers Exposed to Coronavirus Disease 2019. JAMA Netw Open 3(3), e203976.

308 Li, F., Wu, JF., Mai, XH, Ning, K., Chen, KY., Chao, L., Zheng, X., 2016. Internalized Homophobia and

309 Depression in Homosexuals: The Role of Self-concept Clarity (in Chinese). Chinese Journal of Clinical Psychology

310 24(3), 475-479.

311 Li, Z., Ge, J., Yang, M., Feng, J., Qiao, M., Jiang, R., Bi, J., Zhan, G., Xu, X., Wang, L., Zhou, Q., Zhou, C., Pan,

312 Y., Liu, S., Zhang, H., Yang, J., Zhu, B., Hu, Y., Hashimoto, K., Jia, Y., Wang, H., Wang, R., Liu, C., Yang, C.,

313 2020. Vicarious traumatization in the general public, members, and non-members of medical teams aiding in

314 COVID-19 control. Brain, Behavior, and Immunity 88, 916-919.

315 Liang, D., Fan, G., 2020. Social Support and User Characteristics in Online Diabetes Communities: An In-Depth

316 Survey of a Large-Scale Chinese Population. Int J Environ Res Public Health 17(8).

317 Lu, D., Wang, H., Yu, R., Yang, H., Zhao, Y., 2020. Integrated infection control strategy to minimize nosocomial

318 infection of coronavirus disease 2019 among ENT healthcare workers. J Hosp Infect 104(4), 454-455.

319 Ma, Y.-F., Li, W., Deng, H.-B., Wang, L., Wang, Y., Wang, P.-H., Bo, H.-X., Cao, J., Wang, Y., Zhu, L.-Y., Yang,

320 Y., Cheung, T., Ng, C.H., Wu, X., Xiang, Y.-T., 2020. Prevalence of depression and its association with quality of

321 life in clinically stable patients with COVID-19. Journal of Affective Disorders 275, 145-148.

322

Malhi, G.S., Mann, J.J., 2018. Depression. Lancet 392(10161), 2299-2312.

323

324

325

326

327

328

329

330

331

332

333

334

335

336

337

338
Mammen, G., Faulkner, G., 2013. Physical activity and the prevention of depression: a systematic review of prospective studies. Am J Prev Med 45(5), 649-657.

Mathew, A.R., Hogarth, L., Leventhal, A.M., Cook, J.W., Hitsman, B., 2017. Cigarette smoking and depression comorbidity: systematic review and proposed theoretical model. Addiction 112(3), 401-412.

NHS, 2020. Coronavirus: Dr El-Hawrani, the NHS and the death of facts.

https://www.nhs.uk/profiles/consultant/4644578.

Nickell, L.A., Crighton, E.J., Tracy, C.S., Al-Enazy, H., Bolaji, Y., Hanjrah, S., Hussain, A., Makhlouf, S., Upshur, R.E., 2004. Psychosocial effects of SARS on hospital staff: survey of a large tertiary care institution. Cmaj 170(5), 793-798.

Nilan, K., McKeever, T.M., McNeill, A., Raw, M., Murray, R.L., 2019. Prevalence of tobacco use in healthcare workers: A systematic review and meta-analysis. PLoS One 14(7), e0220168.

Pan, X., Xiao, Y., Ren, D., Xu, Z.M., Zhang, Q., Yang, L.Y., Liu, F., Hao, Y.S., Zhao, F., Bai, Y.H., 2020.

Prevalence of mental health problems and associated risk factors among military healthcare workers in specialized COVID-19 hospitals in Wuhan, China: A cross-sectional survey. Asia-Pacific Psychiatry, e12427.

Parisi, J.M., Xia, J., Spira, A.P., Xue, Q.L., Rieger, M.L., Rebok, G.W., Carlson, M.C., 2014. The Association

Between Lifestyle Activities and Late-Life Depressive Symptoms. Act Adapt Aging 38(1), 1-10. 
339 Rakofsky, J.J., Schettler, P.J., Kinkead, B.L., Frank, E., Judd, L.L., Kupfer, D.J., Rush, A.J., Thase, M.E., Yonkers, 340 K.A., Rapaport, M.H., 2013. The prevalence and severity of depressive symptoms along the spectrum of unipolar

341 depressive disorders: a post hoc analysis. The Journal of clinical psychiatry 74(11), 1084-1091.

342 Rep, M.M.M.W., 2003. Cluster of severe acute respiratory syndrome cases among protected health-care workers--

343 Toronto, Canada, April 2003. MMWR Morb Mortal Wkly Rep 52(19), 433-436.

344 Roche, G.C., Fung, P., Ransing, R., Noor, I.M., Shalbafan, M., El Hayek, S., Koh, E.B.Y., Gupta, A.K., Kudva,

345 K.G., 2020. The state of psychiatric research in the Asia Pacific region. Asia-Pacific Psychiatry, e12432.

346 Schneider, A., Bak, M., Mahoney, C., Hoyle, L., Kelly, M., Atherton, I.M., Kyle, R.G., 2019. Health-related

347 behaviours of nurses and other healthcare professionals: A cross-sectional study using the Scottish Health Survey. J

348 Adv Nurs 75(6), 1239-1251.

349 Skevington, S.M., Lotfy, M., O'Connell, K.A., Group, W., 2004. The World Health Organization's WHOQOLBREF quality of life assessment: psychometric properties and results of the international field trial. A report from the WHOQOL group. Qual Life Res 13(2), 299-310.

352 Tran, K., Cimon, K., Severn, M., Pessoa-Silva, C.L., Conly, J., 2012. Aerosol generating procedures and risk of

353 transmission of acute respiratory infections to healthcare workers: a systematic review. PLoS One 7(4), e35797.

354 Venugopal, V.C., Mohan, A., Chennabasappa, L.K., 2020. Status of mental health and its associated factors among the general populace of India during COVID-19 pandemic. Asia-Pacific Psychiatry, e12412.

356 Voruganti, L., Heslegrave, R., Awad, A.G., Seeman, M.V., 1998. Quality of life measurement in schizophrenia:

357 reconciling the quest for subjectivity with the question of reliability. Psychol. Med. 28(1), 165-172.

358

359

360

361

362

363

364

365

366

367

368

369

370

371

372

373

374

375

376

Wang, D., Hu, B., Hu, C., Zhu, F., Liu, X., Zhang, J., Wang, B., Xiang, H., Cheng, Z., Xiong, Y., Zhao, Y., Li, Y., Wang, X., Peng, Z., 2020. Clinical Characteristics of 138 Hospitalized Patients With 2019 Novel CoronavirusInfected Pneumonia in Wuhan, China. JAMA.

Wang, W.-C., Yao, G., Tsai, Y.-J., Wang, J.-D., Hsieh, C.-L., 2006. Validating, Improving Reliability, and Estimating Correlation of the Four Subscales in the WHOQOL-BREF using Multidimensional Rasch Analysis. Quality of Life Research 15(4), 607-620.

Wittkampf, K.A., Naeije, L., Schene, A.H., Huyser, J., van Weert, H.C., 2007. Diagnostic accuracy of the mood module of the Patient Health Questionnaire: a systematic review. Gen Hosp Psychiatry 29(5), 388-395.

Woo, Y.S., Rosenblat, J.D., Kakar, R., Bahk, W.-M., McIntyre, R.S., 2016. Cognitive deficits as a mediator of poor occupational function in remitted major depressive disorder patients. Clinical Psychopharmacology and Neuroscience 14(1), 1 .

World Health Organization, 2020. The Coronavirus disease (COVID-19) outbreak. https://www.who.int (access March 30th 2020).

Xi, X., Liu, YF., 2017. The appliation of Wechat platform and Wenjuanxing in cognitive training among psychiatric nurse, cleaning staff and patients (in Chinese). Nursing Practice and Research 14(21), 114-117.

Xia, P., Li, N., Hau, K.T., Liu, C., Lu, Y., 2012. Quality of life of Chinese urban community residents: a psychometric study of the mainland Chinese version of the WHOQOL-BREF. BMC Med Res Methodol 12, 37.

Xu, J., Xu, Q.H., Wang, C.M., Wang, J., 2020. Psychological status of surgical staff during the COVID-19 outbreak. Psychiatry research 288, 112955.

Peer] reviewing PDF | (2020:12:55976:2:0:NEW 1 Feb 2021) 
377 Yip, P.S., Cheung, Y., Chau, P.H., Law, Y., 2010. The impact of epidemic outbreak: the case of severe acute 378 respiratory syndrome (SARS) and suicide among older adults in Hong Kong. Crisis: The Journal of Crisis

379 Intervention and Suicide Prevention 31(2), 86.

380 Zhang, W.R., Wang, K., Yin, L., Zhao, W.F., Xue, Q., Peng, M., Min, B.Q., Tian, Q., Leng, H.X., Du, J.L., Chang, 381 H., Yang, Y., Li, W., Shangguan, F.F., Yan, T.Y., Dong, H.Q., Han, Y., Wang, Y.P., Cosci, F., Wang, H.X., 2020. 382 Mental Health and Psychosocial Problems of Medical Health Workers during the COVID-19 Epidemic in China.

383 Psychother Psychosom, 1-9.

384 Zhao, S., Lin, Q., Ran, J., Musa, S.S., Yang, G., Wang, W., Lou, Y., Gao, D., Yang, L., He, D., Wang, M.H., 2020. 385 Preliminary estimation of the basic reproduction number of novel coronavirus (2019-nCoV) in China, from 2019 to 2020: A data-driven analysis in the early phase of the outbreak. Int J Infect Dis 92, 214-217.

Zhong, B.L., Luo, W., Li, H.M., Zhang, Q.Q., Liu, X.G., Li, W.T., Li, Y., 2020. Knowledge, attitudes, and practices towards COVID-19 among Chinese residents during the rapid rise period of the COVID-19 outbreak: a quick online cross-sectional survey. Int J Biol Sci 16(10), 1745-1752. 


\section{Table $\mathbf{1}$ (on next page)}

Table 1 Demographic characteristics of the study cohort of ENT nurses 
Table 1 Demographic characteristics of the study cohort of ENT nurses

\begin{tabular}{|c|c|c|c|c|c|c|c|c|c|}
\hline \multirow[t]{2}{*}{ Variables } & \multicolumn{2}{|c|}{$\begin{array}{c}\text { Total } \\
(\mathbf{N}=\mathbf{1 , 7 5 7})\end{array}$} & \multicolumn{2}{|c|}{$\begin{array}{l}\text { No } \\
\text { depression } \\
\quad(N=1,164)\end{array}$} & \multicolumn{2}{|c|}{$\begin{array}{c}\text { Depression } \\
(\mathbf{N}=\mathbf{5 9 3})\end{array}$} & \multirow[t]{2}{*}{$\mathbf{X}^{2}$} & \multirow[t]{2}{*}{ df } & \multirow[t]{2}{*}{$\boldsymbol{P}$} \\
\hline & $\mathbf{N}$ & $\%$ & $\mathbf{N}$ & $\%$ & $\mathbf{N}$ & $\%$ & & & \\
\hline Married & 1310 & 74.6 & 875 & 75.2 & 435 & 73.4 & 0.683 & 1 & 0.409 \\
\hline College education and above & 1707 & 97.2 & 1131 & 97.2 & 576 & 97.1 & 0.001 & 1 & 0.970 \\
\hline Living with family & 1483 & 84.4 & 988 & 84.9 & 495 & 83.5 & 0.590 & 1 & 0.442 \\
\hline Junior rank & 1017 & 57.9 & 683 & 58.7 & 334 & 56.3 & 0.892 & 1 & 0.345 \\
\hline Experience with SARS & 204 & 11.6 & 136 & 11.7 & 68 & 11.5 & 0.018 & 1 & 0.893 \\
\hline Working in tertiary hospitals & 1528 & 87.0 & 1001 & 86.0 & 527 & 88.9 & 2.862 & 1 & 0.091 \\
\hline Working in inpatient department & 1535 & 87.4 & 1024 & 88.0 & 511 & 86.2 & 1.154 & 1 & 0.283 \\
\hline Shift duty & 1195 & 68.0 & 789 & 67.8 & 406 & 68.5 & 0.084 & 1 & 0.772 \\
\hline Local COVID-19 cases $\geq 500$ & 235 & 13.4 & 161 & 13.8 & 74 & 12.5 & 0.620 & 1 & 0.431 \\
\hline $\begin{array}{ll}\text { Having } & \text { infected } \\
\text { family/friends/colleagues } & \end{array}$ & 86 & 4.9 & 50 & 4.3 & 36 & 6.1 & 2.660 & 1 & 0.103 \\
\hline Looking after infected patients & 158 & 9.0 & 92 & 7.9 & 66 & 11.1 & 4.996 & 1 & 0.025 \\
\hline \multirow[t]{2}{*}{ Current smoker } & 13 & 0.7 & 5 & 0.4 & 8 & 1.3 & 4.523 & 1 & $\mathbf{0 . 0 3 3}$ \\
\hline & Mean & SD & Mean & SD & Mean & SD & $\mathbf{T} / \mathbf{Z}$ & df & $\boldsymbol{P}$ \\
\hline Age (years) & 34.09 & 8.03 & 33.86 & 8.23 & 34.56 & 7.62 & -1.729 & $\begin{array}{c}175 \\
5\end{array}$ & 0.084 \\
\hline Work experience (years) & 12.72 & 8.82 & 12.51 & 9.01 & 13.12 & 8.42 & -2.324 & $---\dagger$ & 0.020 \\
\hline Total QOL score & 6.64 & 1.57 & 7.18 & 1.36 & 5.58 & 1.40 & 23.064 & $\begin{array}{c}175 \\
5\end{array}$ & $\begin{array}{c}<0.00 \\
1\end{array}$ \\
\hline
\end{tabular}

Note: due to the very small sample size of male nurses in this sample $(\mathrm{N}=11)$, gender was not included in analyses.

$\uparrow$ : Mann-Whitney U test; Bolded values: P<0.05; M: mean; SD: standard deviation; COVID-19: Corona Virus Disease 2019; SARS:

Severe Acute Respiratory Syndrome; QOL: Quality of Life 
Table 2 (on next page)

Table 2 Independent correlates of depression by multiple logistic regression analysis 
1 Table 2 Independent correlates of depression by multiple logistic regression analysis

\begin{tabular}{lccc}
\hline \multirow{2}{*}{ Variables } & \multicolumn{2}{c}{ Multiple logistic regression analysis } \\
\cline { 2 - 4 } & OR & $\mathbf{9 5 \%}$ CI & P value \\
\hline Working in tertiary hospitals & 1.295 & $0.953-1.761$ & 0.098 \\
Looking after infected patients & 1.441 & $1.031-2.013$ & $\mathbf{0 . 0 3 2}$ \\
Current smoker & 2.880 & $1.018-8.979$ & $\mathbf{0 . 0 4 8}$ \\
Age (years) & 1.028 & $0.984-1.074$ & 0.216 \\
Work experience (years) & 0.984 & $0.945-1.024$ & 0.423 \\
\hline
\end{tabular}

Note: No collinearity between independent variables was found.

Bolded values: $\mathrm{P}<0.05$; CI: confidential interval; OR: odds ratio; QOL: Quality of Life 